\title{
Linear DNA amplicons as a novel cancer vaccine strategy
}

$5 \quad$ Antonella Conforti ${ }^{1,2^{\star}}$, Erika Salvatori ${ }^{1}$, Lucia Lione $^{1}$, Mirco Compagnone ${ }^{3}$, Eleonora

6 Pinto $^{1}$, Clay Shorrock ${ }^{4}$, James A. Hayward ${ }^{4}$, Yuhua Sun $^{4}$, Ben Minghwa Liang ${ }^{4}$, Fabio

Palombo $^{1,3^{* *}}$, Brian Viscount ${ }^{* *}$ and Luigi Aurisicchio ${ }^{1,2,3^{*}}$

10 1Takis Biotech, Via Castel Romano 100, 00128 Rome, Italy

11 2Evvivax Biotech, Via Castel Romano 100, 00128 Rome, Italy

$12{ }^{3}$ Neomatrix Biotech, Via Castel Romano 100, 00128 Rome, Italy

$13 \quad{ }^{4}$ Applied DNA Sciences, 50 Health Sciences Drive, Stony Brook, NY 11790

14

15 Text word count: 4059

16 Reference count: 32

17 Table/Figure: 5

18

$19{ }^{*}$ Corresponding authors: Antonella Conforti (conforti@evvivax.com) or Luigi Aurisicchio

20 (aurisicchio@evvivax.com)

$22{ }^{* \star}$ Co-corresponding authors: Brian Viscount (brian.viscount@adnas.com) or Fabio

23 Palombo (palombo@neomatrixbiotech.com) 


\section{Abstract}

26 DNA-based vaccines represent a simple, safe and promising strategy for harnessing the

27 immune system to fight infectious diseases as well as various forms of cancer and thus

28 are considered an important tool in the cancer immunotherapy toolbox. Nonetheless, the

29 manufacture of plasmid DNA vaccines has several drawbacks, including long lead times

30 and the need to remove impurities from bacterial cultures. Here we report the

31 development of polymerase chain reaction (PCR)-produced amplicon expression vectors

32 as DNA vaccines and their in vivo application to elicit antigen-specific immune responses

33 in animal cancer models. Amplicons encoding tumor-associated-antigens, such as

34 telomerase reverse transcriptase or neoantigens expressed by murine tumor cell lines

35 were able to elicit antigen-specific immune responses and proved to significantly impact

36 tumor growth when administered in combination with immune-checkpoint inhibitors (ICls).

37 These results strongly support the further exploration of the use of PCR-based amplicons

38 as an innovative immunotherapeutic approach to cancer treatment.

\section{Keywords}

43 DNA vaccine, PCR, amplicons, cancer immunotherapy. 


\section{Introduction}

48 Cancer is the leading cause of mortality worldwide. Conventional therapies such as

49 surgery, radiation and chemotherapy, are considered aggressive and can negatively

50 impact the patient without offering lifelong protection, immunotherapy has in recent years

51 emerged as a promising, more tolerable and more durable form of cancer treatment.

52 Among the newest immunotherapeutic approaches to cancer treatment, DNA-based

53 cancer vaccines are important strategies in the cancer immunotherapy toolbox. ${ }^{1}$ DNA

54 vaccines for cancer immunotherapy are designed to deliver one or several genes

55 encoding tumor-associated antigens or other immunogenic polypeptides to modulate

56 immune responses, thereby eliciting or strengthening immune responses against tumor

57 antigens that play a central role in tumor initiation, progression and metastasis, or arise

58 as a result of mutational burden, e.g. neoantigens. ${ }^{2,3,4,5}$ DNA cancer vaccines can induce

59 both innate immunity and adaptive immune responses which can suppress tumor growth

60 and, in some cases, achieve total tumor rejection or shrinkage.6,7,8,9 To date, DNA based

61 vaccines have involved the inoculation of a subject with plasmid DNA and/or plasmid-

62 derived DNA. Bacterial plasmids are episomal circular DNA constructs propagated in

63 bacteria via a fermentation process. However, the manufacture and use of DNA vaccines

64 via plasmids has several drawbacks, including the presence of antibiotic resistance

65 genes, the presence of unwanted additional DNA sequences in the form of the plasmid

66 backbone, and the need to remove impurities from bacterial cultures, such as bioburden

67 and endotoxins, long lead times, inefficient uptake of the large plasmid DNA molecules

68 to the cellular nucleus and challenges of integrating plasmid production into automated

69 cGMP (current Good Manufacturing Practices) workflows. Thus, there is an unmet need 
70 for a new DNA vaccination platform not including bacterial plasmids or plasmid-derived

71 DNA. In this study, we report the development of an innovative DNA vaccine based on a

72 DNA amplicon produced by the enzymatic amplification process of an expression vector

73 via the polymerase chain reaction (PCR). In order to improve the weak immunogenicity

74 induced by naked DNA injection, we exploited electroporation (EP) as a powerful delivery

75 technology, considering its safety, low cost, efficacy and ease of application. This

76 procedure consists of exposing a cell or a tissue to an external electric field that increases

77 cell membrane permeability to molecules that otherwise would cross the plasma

78 membrane with low efficiency; for this reason, it has been widely used in different

79 biomedical applications. ${ }^{10,11,12}$ Comparison between DNA injection alone and DNA-EP

80 has demonstrated an increase in both cellular and humoral response after electric fields

81 were applied. It has been demonstrated that the addition of electroporation provides a 10-

82 100- fold augmentation of immune response and defense against pathogens in humans

83 and numerous animal models of diseases such as HIV/SIV, malaria, hepatitis B and C,

84 human papilloma virus (HPV), anthrax and influenza. ${ }^{13}$ Furthermore, EP may enhance

85 immune responses through increased protein expression, secretion of inflammatory

86 chemokines and cytokines, and recruitment of monocytes and antigen-presenting cells at

87 the site of electroporation. ${ }^{14,15}$ In addition, in vivo electroporation causes transient and

88 reversible cell damage also resulting in local inflammation and release of cytokines which

89 may act as a danger signal, consequently enhancing vaccine potency. ${ }^{16,17}$ Here, we

90 demonstrate gene expression mediated by DNA amplicons both in vitro and in vivo and

91 show the immunogenicity of a DNA amplicon encoding for a tumor associated antigen

92 (i.e. Telomerase Reverse Transcriptase - TERT) in wild-type mice as compared to 
93 plasmid DNA-EP. Furthermore, we show that DNA amplicons encoding neoantigens and

94 delivered by EP induce therapeutic effects in tumor-bearing mice, when combined with

95 Immune Checkpoint Inhibitors (ICls), in comparison to the combination of plasmid DNA-

96 EP and ICls.

97

98

99 Materials and Methods

100

$101 \quad$ Plasmid DNA constructs

102 All constructs were completely synthesized and optimized for codon usage.

103 Synthesis and codon optimization analysis of plasmid DNA vectors were performed at

104 Genscript (China).

105 Luciferase gene, cloned into the linearized pcDNA3-Hygro vector by enzymatic

106 restriction, was used to assess gene expression both in vitro (by transfecting cells) and

107 in vivo (by mice immunization).

108 conTRT vaccine consisted of a DNA plasmid encoding a consensus sequence between

109 canine and human telomerase. In addition, this catalytically inactive telomerase protein

110 has been fused to a TPA (human tissue plasminogen activator) leader sequence at $\mathrm{N}$ -

111 term and fused itself to the Profilin-like sequence of Toxoplasma gondii (PFTG) at the C-

112 terminus (TPA-conTRT-PFTGopt). This construct was finally cloned into the linearized

113 pTK1A-TPA vector by enzymatic restriction. Neoantigen-based vaccines are DNA

114 plasmids encoding for neoepitopes selected from a murine colon adenocarcinoma cell

115 line (i.e., MC38 cell line, ATCC, USA). Once identified by RNA sequencing, genes 
116 encoding for selected neoepitopes were cloned in pTK1A vector, as previously

117 described. 7,18

119 DNA Amplicon Constructs

120 PCR primers were designed to amplify the expression cassette contained in the plasmid

121 sequences for the Luciferase, conTRT vaccine and Neoantigen-based vaccine (M8)

122 constructs, so creating dsDNA amplicons consisting only of the expression cassette

123 sequence (namely, DNA amplicon expression vector). For the phosphothiate-modified

124 amplicons, a sulfur atom substitutes the non-bridging oxygen in the phosphate backbone

125 of the oligonucleotide. In addition, the three (3') terminal bases of both the forward and 126 reverse primers were modified to increase DNA amplicon stability. ${ }^{19}$ DNA amplifications

127 via PCR for the Luciferase constructs were performed using GeneAmp 2700 PCR system

128 (Applied Biosystem, USA) using either BIOLASETM polymerase $\left(7 \times 10^{-5}\right.$ approximate 129 error/bp) or MyFiTM polymerase (7×10-5 approximate error/bp) both from Bioline (Meridian

130 Bioscience, USA). The PCR mixture consisted of $1 \mathrm{X}$ buffer with $1 \mathrm{mM}$ dNTP, $0.5 \mu \mathrm{M}$ of

131 forward and reverse primers, polymerase $5 \mathrm{u} / 100 \mathrm{ul}$, and plasmid template at $40 \mathrm{ng} / \mathrm{mL}$.

132 The PCR reactions were subjected to initial denaturation of $97^{\circ} \mathrm{C}$ for 20 seconds, and

133 subsequent 30 cycles of $95^{\circ} \mathrm{C}$ for 10 seconds and $72^{\circ} \mathrm{C}$ for 5 minutes followed by final

134 extension at $72^{\circ} \mathrm{C}$ for 3 minutes. Four different versions of Luciferases DNA amplicon

135 expression vectors were produced using either regular primers or phosphothiate-modified

136 primers using either the Biolase or MyFi polymerase, resulting in 2878 bp DNA amplicon

137 expression vectors. DNA amplifications for the conTRT amplicons were performed

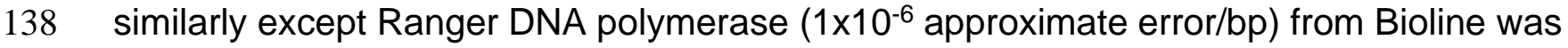


139 utilized, resulting in 6024 bp DNA amplicon expression vectors. DNA amplifications for

140 the M8 amplicon were also performed similarly except Q5 ${ }^{\mathrm{TM}}$ polymerase $\left(5.3 \times 10^{-7}\right.$

141 approximate error/bp; New England BioLabs, USA) was utilized, resulting in 3037 bp DNA

142 amplicon expression vectors.

\section{DNA purification and qualification}

146 Each dsDNA amplicon expression vector produced via the PCR amplification was purified

147 on an Akta Pure 150 FPLC instrument (GE Healthcare, USA). After the entire lot was

148 loaded onto the GE ReadyToProcess Adsorber Q anion exchange column and washed,

149 a linear gradient of up to $1.5 \mathrm{M} \mathrm{NaCl}$ in $1 \times$ PBS was used to elute DNA via $0.5 \mathrm{~mL}$

150 fractions. These fractions were analyzed with DNA 7500 assay on 2100 Bioanalyzer

151 (Agilent, USA). Fractions containing the DNA amplicon expression vectors of interest

152 were identified, pooled, and concentrated using ethanol precipitation and refrigerated

153 centrifugation. After rinsing and drying, each DNA amplicon expression vector was

154 resuspended to the desired concentration in 1x PBS, and sterile filtered. Further analytical

155 characterization of each quantity of DNA amplicon expression vector was performed

156 using NanoDrop (Thermo Fisher, USA), 2100 Bioanalyzer, and Alliance HPLC System

157 (Waters, USA).

158 Based on the above workflows, the following DNA expression vectors (DNA amplicons)

159 were produced: as for Luciferase, \#349 (PS-modified; MyFi Polymerase), \#351 (PS-

160 modified; Biolase Polymerase), \#355 (MyFi Polymerase), \#358 (Biolase Polymerase); as

161 for conTRT, \#359 (PS-modified; Ranger Polymerase), \#360 (PS-modified; Biolase 
162 Polymerase), \#363 (Ranger Polymerase), \#365 (Biolase Polymerase); as for M8, \#12290

163 (Q5 Polymerase).

164

165 Vaccination and murine models

166 For in vivo expression and immunogenicity assessment, 6-8 weeks old BALB/c female

167 mice (Envigo, USA) were injected intramuscularly (i.m.), particularly in the quadriceps,

168 with either DNA plasmid or amplicon (dose ranging from $5 \mu \mathrm{g}$ to $50 \mu \mathrm{g}$ ) and electrically

169 stimulated as previously described. ${ }^{20,21}$ The DNA was formulated in Phosphate Buffered

170 Saline (PBS) at a concentration of $0.1-1 \mathrm{mg} / \mathrm{ml}$. DNA-EP was performed by means of a

171 Cliniporator Device EPS01 and using N-10-4B electrodes (IGEA, Italy) with the following

172 electrical conditions in Electro-Gene-Transfer (EGT) modality: 8 pulses 20 msec each at

$173110 \mathrm{~V}, 8 \mathrm{~Hz}, 120 \mathrm{msec}$ interval. As for the evaluation of the antitumoral efficacy of plasmid

174 and amplicon DNA in cancer models, tumor challenge was performed by injecting $3 \times 10^{5}$

175 MC38 cells subcutaneously in the right flank of $6-8$ weeks old female C57BI/6 mice

176 (Envigo). Twice a week, mice were examined for tumor growth using a caliper. Tumor

177 volumes were calculated using the formula:

$$
\frac{\text { Lengh } \times W i d t h^{\wedge} 2}{2}
$$

179 Mice were housed according to national legislation and kept in standard conditions

180 according to Evvivax ethical committee approval. All the in vivo experimental procedures

181 were approved by the local animal ethics council.

182

183 Peptides 
184 Lyophilized peptides covering telomerase reverse transcriptase protein or neoepitopes

185 were purchased from JPT (Germany) and resuspended in DMSO at $40 \mathrm{mg} / \mathrm{ml}$. As for

186 telomerase, pools of peptides of 15 aa overlapping by 11 residues were assembled in

187 four pools (A, B, C, D). Peptides and pools were stored at $-80^{\circ} \mathrm{C}$.

189 Luciferase assay

190 In vitro luciferase assay was performed by cell transfection. Briefly, Hela cells were plated

191 at $1 \times 10^{5} /$ well in 96 well plate and then transfected with equimolar concentrations of DNA

192 plasmid or DNA amplicon (1 and $0.3 \mu \mathrm{g}$ ). After 24 hours, luciferase expression signal was

193 measured by Imaging at an IVIS 200 imaging device (Perkin Elmer, USA).

194 In order to assess luciferase expression in vivo, female BALB/c mice (5 mice/group) were

195 anesthetized using 97\% oxygen and $3 \%$ isoflurane (Isoba, UK) then injected by EP in

196 quadriceps muscle with a DNA plasmid encoding Luciferase (pcDNA3-Hygro-Luc at 1

$197 \mu \mathrm{g} /$ mouse) or equimolar concentrations of DNA amplicon. Imaging was performed under 198 gas anesthesia at IVIS 200 imaging system at 24h, 48h and one week after injection, 8

199 min after injecting subcutaneously a luciferin substrate solution (15mg/ml, Perkin Elmer)

200 at $10 \mu \mathrm{l} / \mathrm{g}$ of body weight.

201

202 Flow Cytometry analysis

203 In experiments performed with vaccinated mice, the intracellular staining was 204 performed according to the procedure described in Giannetti et al. ${ }^{22}$ Briefly, PBMC or 205 splenocytes were treated with ACK Lysing buffer (Life Technologies, USA) for red blood 206 cell lysis and resuspended in $0.6 \mathrm{ml} \mathrm{RPMI}, 10 \%$ FCS and incubated with the indicated 
207 pool of peptides $(5 \mu \mathrm{g} / \mathrm{ml}$ final concentration of each pool) and brefeldin A ( $1 \mu \mathrm{g} / \mathrm{ml}$; BD

208 Pharmingen, USA) at $37^{\circ} \mathrm{C}$ for $12-16$ hours. Cells were then washed and stained with

209 surface antibodies. After washing, cells were fixed, permeabilized and incubated with anti-

210 IFN $\gamma\left(X_{M G 1.2)}\right.$ and anti-TNF $\alpha$ (MP6-XT22; all from eBioscience, USA), fixed with 1\%

211 formaldehyde in PBS, acquired by means of a CytoFLEX LX flow cytometer (Beckman

212 Coulter, USA) and analysis was performed using CytExpert software (Beckman Coulter).

213 DMSO and PMA/IONO (Sigma, Italy) at $10 \mu \mathrm{g} / \mathrm{ml}$ were used as internal negative and 214 positive control of the assay, respectively.

$216 \underline{\text { IFN }}$ ELISpot

217 T cell ELISpot for mouse IFN $\gamma$ was performed as previously described..$^{23}$ Briefly, 218 splenocytes isolated from either conTRT vaccinated BALB/c mice or neoantigen 219 vaccinated mice were stimulated for $20 \mathrm{~h}$ with telomerase or neoantigen peptide pools (1 $220 \mu \mathrm{g} / \mathrm{ml}$ as final concentration). After developing the assay, spot forming cells (SFCs) were 221 counted using an automated ELISPOT reader (A.EL.VIS ELIspot reader, Germany).

\section{Statistical analyses}

$224 \quad$ Statistical analyses were performed with GraphPad Prism software version 8 225 (GraphPad). $n$ represents individual mice analyzed per experiments. Error bars indicate 226 the standard error of the mean (SEM). We used Mann-Whitney U-tests to compare two 227 groups with non-normally distributed continuous variables. Significance is indicated as 228 follows: ${ }^{*} p<0.05 ;{ }^{* *} p<0.01$. Comparisons are not statistically significant unless indicated. 


\section{$230 \quad$ Results}

\section{Manufacturing of DNA Amplicons}

232 A schematic representation showing the assembly of termini phosphorothioate-modified

233 (PS-modified) amplicon expression vectors via a PCR device is shown (Fig. 1A). The

234 DNA sequence of the amplicon expression vector (taken from plasmid DNA expression

235 cassette sequence) includes a promoter, one open reading frame (ORF) and a terminator.

236 It may optionally include a fusion ORF or other secondary ORFs and/or one or more

237 enhancers. Once the sequence is known, a template amplicon expression vector is

238 created via DNA synthesis, without the use of plasmid DNA. Appropriate PS-modified

239 PCR primers for the specific sequence of the template amplicon expression vector are

240 generated via oligonucleotide synthesis. The template amplicon expression vector and

241 the amine-modified primers are introduced to a PCR device whereby the template

242 amplicon expression vector is exponentially amplified via PCR to create amplicon

243 expression vector with PS-modified 3' and/or 5' termini. Upon completion of the PCR

244 reaction the amplicon expression vectors are purified and ready for use in the transfection

245 of a target cell for expression of the desired peptide, antigen, polypeptide or protein.

246 As shown in Fig.1B, for the luciferase expression cassette, human CMV promoter drives

247 the expression of firefly luciferase and a bovine growth hormone $(\mathrm{bGH})$ poly $\mathrm{A}$ has been

248 used as termination site. conTRT is a codon-optimized, consensus telomerase fused with

249 Profilin-like from Toxoplasma Gondii (PFTG). M8 is a polypeptide encoding 8 neoantigens

250 expressed by MC38 murine colon cancer cells. ${ }^{5,7}$ For conTRT and M8 constructs, a

251 human CMV promoter/intron A drives gene expression as well. 
252 Various polymerases, with different error rates, have been used for the PCR-based

253 production of the amplicon expression vectors. BIOLASETM polymerase $\left(7 \times 10^{-5}\right.$

254 approximate error/bp), MyFi ${ }^{\mathrm{TM}}$ polymerase (7x10-5 approximate error/bp) and RANGER ${ }^{\mathrm{TM}}$

255 polymerase $\left(1 \times 10^{-6}\right.$ approximate error/bp) have been used to amplify the expression

256 cassette in the presence or absence of PS-modified primers. Figures 1C and 1D show

257 the purity of the amplicons encoding luciferase, measured by HPLC and gel

258 electrophoresis, respectively. These data confirm that the usage of different Taq

259 polymerase, in the presence of PS-modified primers or not, does not affect DNA 260 amplicons purity.

262 Assessment of in vitro and in vivo expression of plasmid and DNA amplicons.

263 In order to compare the expression capacity of DNA amplicons to plasmid DNA 264 expression, the four differentially manufactured PCR products were evaluated by means 265 of in vitro cell transfection and in vivo injection. For in vitro assay, Hela cells were 266 transfected with equimolar concentrations of plasmid DNA (pcDNA3-Hygro-Luc) and four 267 different PCR products (1 and $0.3 \mu \mathrm{g}$ dose), all encoding luciferase. 24 hours after 268 transfection, luciferase signal was revealed both in plasmid DNA and in DNA amplicon 269 transfected cells, at all tested doses, although at lower extent in latter ones (data not 270 shown). For in vivo evaluation of luciferase expression, BALB/c mice were injected i.m. 271 by DNA-EP, with equimolar concentrations of plasmid DNA or DNA amplicons encoding 272 for luciferase (50-10-5 $\mu \mathrm{g} /$ mouse). Luciferase signal was measured by performing optical 273 imaging 24h, 48h and one week post injection (Fig.2A). As shown in Fig.2B, all mice 274 showed luciferase expression in both legs with $50 \mu \mathrm{g}$ plasmid DNA dose (corresponding 
275 to $18.75 \mu \mathrm{g}$ of DNA amplicon) at each evaluation time point, despite a consistent decrease

276 in mice treated with DNA amplicons at one week post injection. Conversely, mice treated

277 with $50 \mu \mathrm{g}$ of plasmid DNA showed a constantly high luciferase expression. Analysis of

278 counts values confirmed the expression capacity of luciferase-encoding PCR products,

279 although lower than that of plasmid DNA. Interestingly, while luciferase expression by

280 plasmid DNA kept growing from 24h measurement to one week after injection, luciferase

281 signal in mice treated with DNA amplicons reached the highest value at 48h post injection

282 and declined at one week time point, although still being measurable (Fig. 3A). This

283 observation was generally valid also in mice injected with $10 \mu \mathrm{g}$ of plasmid DNA

284 (corresponding to $3.75 \mu \mathrm{g}$ of DNA amplicon), although with greater variability (Fig.3B),

285 and in mice injected with $5 \mu \mathrm{g}$ of DNA amplicon (corresponding to $1.875 \mu \mathrm{g}$ of PCR

286 product) although with lower counts measured in mice injected with DNA amplicons

287 (Fig.3C). These results show that DNA amplicon expression is stable for at least $48 \mathrm{~h}$ after

288 being injected in mouse quadriceps, being still detectable even at one week after 289 injection.

291 In vivo analysis of T cell response elicited by DNA amplicons.

292 Once we demonstrated the expression of DNA amplicons after in vivo injection, we sought

293 to evaluate their capacity to elicit an immune response against a tumor associated

294 antigen. To this aim, we vaccinated BALB/c mice i.m. by means of DNA-EP, with three

295 doses (50-10-5 $\mu \mathrm{g} /$ mouse) of a DNA plasmid encoding for conTRT. Likewise, we

296 vaccinated BALB/c mice with equimolar doses (33.75-6.75-3.375 $\mu \mathrm{g} / \mathrm{mouse})$ of a DNA

297 amplicon encoding for the same sequence. All mice were vaccinated following a prime- 
298 boost regimen (days 0 and 21, see Fig. 4 A) and blood analysis was performed at day 28

299 by intracellular cytokine staining. Cytokine analyses revealed a Th1-skewed immune

300 response, both in plasmid DNA vaccinated mice and DNA amplicon vaccinated mice. For

301 CD8+ T cell response (Fig.4B), there was no significant difference in the magnitude of

302 antigen-specific T cells, at all tested DNA doses, with the only exceptions of mice treated

303 with $10 \mu \mathrm{g}$ of plasmid DNA and mice treated with $5 \mu \mathrm{g}$ of amplicon \#360. As for CD4+ T

304 cell compartment, no significant differences were revealed between plasmid DNA and

305 DNA amplicons vaccinated mice as well, with the only exception of mice treated with $10 \mu \mathrm{g}$

306 of plasmid DNA (Fig.4C). These results demonstrate that, although in some cases there

307 is no dose-dependent immunogenicity, DNA plasmid and PCR product induce

308 comparable immune response in vivo against a tumor associated antigen.

310 Antitumoral effect of DNA amplicons in murine cancer model.

311 After proving that DNA amplicons are able to elicit antigen-specific immune responses as

312 efficiently as plasmid DNA, we investigated whether this novel immunotherapeutic

313 strategy could exert an antitumoral effect in a cancer murine model. To this aim, we

314 exploited a murine cancer model previously described, ${ }^{5}$ where a neoantigen-based DNA

315 vaccine (namely, M8) was shown to induce tumor regression when administered in

316 combination with ICls such as $\alpha$ PD1 and $\alpha \mathrm{CTLA} 4$, in a therapeutic setting. Briefly, as

317 shown in Fig.5A, after tumor challenge, C57BI/6 mice received a combination of DNA

318 (plasmid or amplicon, administered at days 2,9 and 16) and ICls ( $\alpha$ CTLA-4 or $\alpha$ PD1,

319 administered at days 3,6 and 9). Neoantigen-specific T cell response, assessed by IFN $\gamma$

320 ELISpot performed on splenocytes collected at day 23, was significantly increased over 
321 the control mice after cotreatment with plasmid M8 and $\alpha$ CTLA-4 or both ICls, but not in

322 mice cotreated with plasmid M8 and $\alpha$ PD1, thus suggesting a synergistic effect of $\alpha C T L A-$

3234 and plasmid M8 vaccine in eliciting an antitumoral effect in this cancer model.

324 Interestingly, this synergistic effect was confirmed when M8 amplicon was administered

325 in combination with $\alpha$ CTLA-4 (Fig. 5B). Furthermore, the cotreatment of M8 amplicon and

326 both ICls was significantly more efficient in eliciting a neoantigen-specific T cell response

327 than the cotreatment of plasmid M8 and both ICls. This observation was confirmed by the

328 assessment of tumor growth (Fig.5C), measured until day of sacrifice (day 23). As

329 observed in mice cotreated with plasmid M8 and ICIs, also the cotreatment with M8

330 amplicon and ICls was equally effective in slowing down tumor progression over control

331 mice. These data further support the potential use of linear DNA as alternative to plasmid

332 DNA in developing immunotherapeutic strategies against cancer disease.

\section{Discussion}

335 The concept of DNA-based vaccines is an appealing immunotherapeutic approach in

336 many diverse fields, potentially translatable to the clinics, thanks to several beneficial

337 features. In fact, DNA vaccines are engineered for maximal gene expression and

338 immunogenicity and they allow for fast and scalable manufacturing as well as long-term

339 stability at room temperature. Moreover, DNA vaccines do not require complex

340 formulations such as those based on nanoparticles (necessary for peptide- or RNA-based

341 vaccines) and have shown a highly satisfactory safety profile, without the potential risk of

342 integration or pathogenicity. ${ }^{24}$ Importantly, they can be quickly designed from new genetic

343 viral sequences and this technical advantage may be essential not only to respond to the 
344 emergency outbreak of an epidemic ${ }^{25,26}$ but also to generate personalized vaccines for

345 treating tumors. ${ }^{27}$ In order to gain an efficient DNA uptake, new delivery technologies may

346 accelerate the rapidly growing sector of genetic cancer vaccines. Among others,

347 electroporation significantly increases the initial uptake of DNA by local cells and for this

348 reason it has been widely used in different biomedical applications. ${ }^{11}$

349 Nonetheless, one huge hurdle of DNA vaccines manufacturing is their production as

350 plasmids, grown in genetically modified bacteria containing, besides the gene of interest,

351 a bacterial origin of replication and a selective gene, normally encoding for an antibiotic

352 resistance, in order to maintain the persistence of the plasmid in the bacterium. Given the

353 excessive and often inappropriate use of antibiotics, both in human and veterinary

354 medicine, as well as in animal husbandry and agriculture, the last decades have

355 witnessed the spread of these compounds in the environment on a global scale and

356 consequently the onset of multidrug-resistant bacterial pathogens with evident and

357 worrying effects on public health. ${ }^{28,29}$ In response to recent concerns about the antibiotic

358 resistance spread associated with conventional DNA vaccines, alternative technologies

359 have been proposed and new DNA vaccine technologies have recently emerged in which

360 the DNA is manufactured in a cell-free process that avoids bacterial fermentation and

361 yields a vaccine that is structurally linear, such as minicircles based on closed linear

362 DNA. $^{30}$ These new bacteria-free manufacturing platforms have already proven to be

363 stable and immunogenic in preclinical models. ${ }^{31,32}$

364 Here we describe, for the first time in literature, the use of an innovative cell-free

365 manufacturing platform as cancer immunotherapy in preclinical tumor model. In this

366 study, enzymatically produced amplicon expression vectors have been specifically used 
367 as a DNA cancer vaccine to express tumor associated or mutated antigens able to elicit

368 a specific immune response. As a proof of concept, a "universal" cancer DNA vaccine

369 was produced comprising an amplicon expression vector encoding an optimized version

370 of telomerase reverse transcriptase (TERT). High levels of TERT are detected in over

$37185 \%$ of cancers. TERT expression correlates with telomerase activity, which is required

372 for tumor survival and unlimited proliferative capacity of cancer cells. In addition, we

373 explore a personalized approach, i.e. DNA vaccines targeting tumor neoantigens.

374 Neoantigens arise from somatic mutations that differ from wild-type antigens and are

375 specific to each individual subject, or a sub-population of subjects, which provide tumor

376 specific antigen targets. Neoantigens are found only in tumor cells, and thus are not

377 subject to self-tolerance, have a decreased risk of generated autoimmunity and on-target

378 off-tumor effects. Tumor neoantigens may be identified by differential sequencing of a

379 subject's tumor versus wild-type samples, using exome/genome sequences and RNAseq

380 analysis, and the assistance of artificial intelligence, machine learning and predictive

381 algorithms. Through this method, a DNA cancer vaccine comprising one or more

382 amplicon expression vectors encoding the identified neoantigens can be designed and

383 produced that will elicit an antigen-specific immune response to said neoantigens,

384 resulting in a cancer vaccine with limited off-tumor side effects and high efficacy. Such

385 "personalized" neoantigen DNA cancer vaccines are well suited for amplicon expression

386 vectors and their method of rapid manufacture. With modern next generation sequencing

387 (e.g. Illumina NovaSeq 6000), efficient bioinformatics platforms and rapid high-fidelity

388 synthetic DNA synthesis, is it possible to create a therapeutic dose of personalized

389 neoantigen encoding amplicon expression vectors in under 48 hours. 
390 In a first phase, we looked at levels and length of gene expression, using luciferase as

391 biomarker. As described in Fig. 2 and 3, the PCR-produced amplicons are highly stable

392 in vivo over time, keeping a constant luciferase expression level up to 48 hours, then their

393 expression capacity starts declining, although expression may be still detectable one

394 week post injection. In addition, through the incorporation of chemical and/or peptide

395 modifications, PCR-produced amplicons can be optimized for high-level expression within

396 target cells leading to an enhanced antigen-specific immune response. In this

397 perspective, it has been shown here that vaccination of mice with DNA amplicons induces

398 similar levels of antigen-specific $\mathrm{T}$ cell response as compared with plasmid DNA.

399 Particularly, as previously observed, ${ }^{33}$ a strong polyfunctional cell-mediated immune

400 response was elicited by amplicons (Fig.4), as well as plasmids, thus validating this

401 innovative technology as a valid alternative to DNA plasmids in the context of genetic

402 vaccines.

403 Moreover, besides showing strong in vivo stability and immunogenicity, DNA amplicons

404 have been shown to induce a robust antitumoral immune response specific for 405 neoantigens expressed by a colon cancer model. It has been already shown elsewhere

406 that neoantigen-based DNA vaccine approach is a potential alternative to currently 407 established cancer immunotherapies, especially when administered as cotreatment with 408 immune checkpoint inhibitors. ${ }^{5}$ By demonstrating that DNA amplicons encoding for 409 neoantigens are equally effective as plasmids in antitumoral cotreatment with ICls (Fig.5),

410 we here validate the use of amplicon expression vectors as DNA cancer vaccines, as a

411 more cost- and time-effective alternative to conventional plasmids, given their enhanced 412 ability to rapidly manufacture tumor-specific cancer vaccines able to elicit antigen-specific 
413 immune responses with increased efficacy and reduced on-target off-tumor effects.

414 Taken together, these results provide the first in vivo preclinical demonstration of linear

415 DNA amplicons antitumoral efficacy thus encouraging further studies for clinical

416 applications.

419 Acknowledgments

$420 \quad$ Research activities are supported in part by the Italian Ministry of Economic 421 Development through grant $F / 190180 / 01 / X 44$ and Campania Regional grant 422 B61G18000470007.

\section{Author contributions}

424 A.C., E.S., L.L., M.C., E.P. performed experiments; A.C., F.P and L.A. analyzed 425 and interpreted data; A.C. prepared the figures; L.A. provided funding, conceptual advice 426 and edited the manuscript; A.C. coordinated the study and wrote the paper; B.V., Y.S. 427 and B.L. produced the amplicons; J.H. and C.S. provided conceptual advice, coordinated 428 the study and edited the manuscript.

\section{Competing interests}

431 Evvivax, Takis and NeoMatrix are currently developing proprietary nucleic-acid vaccines 432 based on DNA-EP. Applied DNA is commercializing LinearDNA ${ }^{\mathrm{TM}}$, its proprietary, large433 scale polymerase chain reaction ("PCR")-based manufacturing platform that allows for 434 the large-scale production of specific DNA sequences. The Company's common stock is 435 listed on NASDAQ under ticker symbol 'APDN,' and its publicly traded warrants are listed 436 on OTC under ticker symbol 'APPDW.'

439 All data and materials are available in the main text. 
1. Liu, M.A. (2011). DNA vaccines: an historical perspective and view to the future. Immunol. Rev. 239, 62-84.

2. Buonaguro, L., Petrizzo, A., Tornesello, M.L., and Buonaguro, F.M. (2010). Translating tumor antigens into cancer vaccines. Clin. Vaccine Immunol. 18, 2334.

3. Aurisicchio, L., Pallocca, M., Ciliberto, G., and Palombo, F. (2018). The perfect personalized cancer therapy: cancer vaccines against neoantigens. J. Exp. Clin. Cancer Res. 37, 86.

4. Conforti A., Palombo F., and Aurisicchio L. (2022) DNA/RNA based vaccines. In Cancer vaccines as Immunotherapy of Cancer, L. Buonaguro and S. Van der Burg, eds. Elsevier. In press.

5. Salvatori, E., Lione, L., Compagnone, M., Pinto, E., Conforti, A., Ciliberto, G., Aurisicchio, L., and Palombo, F. (2022). Neoantigen cancer vaccine augments anti-CTLA-4 efficacy. npj Vaccines. 7, 15.

6. Aurisicchio, L., and Ciliberto, G. (2012). Genetic cancer vaccines: current status and perspectives. Expert. Opin. Biol. Ther.12, 1043-1058.

7. Aurisicchio, L., Salvatori, E., Lione, L., Bandini, S., Pallocca, M., Maggio, R., Fanciulli, M., De Nicola, F., Goeman, F., Ciliberto, G., et al. (2019). Poly-specific neoantigen-targeted cancer vaccines delay patient derived tumor growth. J. Exp. Clin. Cancer Res. 38, 78.

8. Duperret, E.K., Perales-Puchalt, A., Stoltz, R., Hiranjith, G.H., Mandloi, N., Barlow, J., Chaudhuri, A., Sardesai, N.Y., and Weiner, D.B. (2019). A Synthetic DNA, MultiNeoantigen Vaccine Drives Predominately MHC Class I CD8+ T-cell Responses, Impacting Tumor Challenge. Cancer Immunol. Res. 7, 174-182.

9. Ho, W., Gao, M., Li, F., Li, Z., Zhang, X., and Xu, X. (2021). Next-Generation Vaccines: Nanoparticle-Mediated DNA and mRNA Delivery. Adv. Healthc Mater. 10, e2001812. 
10. Kotnik, T., Frey, W., Sack, M., Meglič, S.H., Peterka, M., and Miklavčič, M. (2015). Electroporation-based applications in biotechnology. Trends Biotechnol. 33, 480488.

11. Lambricht, L., Lopes, A., Kos, S., Sersa, G., Préat, V., and Vandermeulen, G. (2016). Clinical potential of electroporation for gene therapy and DNA vaccine delivery. Expert Opin. Drug Deliv. 13, 295-310.

12. Lione, L., Salvatori, E., Petrazzuolo, A., Massacci, A., Maggio, R., Conforti, A., Compagnone, M., Aurisicchio, L., Ciliberto, G., and Palombo, F. (2021). Antitumor efficacy of a neoantigen cancer vaccine delivered by electroporation is influenced by microbiota composition. Oncoimmunology. 10, 1898832.

13. Heller, R., and Heller, L.C. (2015). Gene electrotransfer clinical trials. Adv. Genet. 89, 235-262.

14.Babiuk, S., Baca-Estrada, M.E., Foldvari, M., Middleton, D.M., Rabussay, D., Widera, G., and Babiuk, L.A. (2004). Increased gene expression and inflammatory cell infiltration caused by electroporation are both important for improving the efficacy of DNA vaccines. J. Biotechnol. 110,1-10.

15. Liu, J., Kjeken, R., Mathiesen, I., and Barouch, D.H. (2008). Recruitment of antigen-presenting cells to the site of inoculation and augmentation of human immunodeficiency virus type 1 DNA vaccine immunogenicity by in vivo electroporation. J. Virol. 82, 5643-5649.

16. Fattori, E., La Monica, N., Ciliberto, G., and Toniatti, C. (2002). Electro-GeneTransfer: A New Approach for Muscle Gene Delivery. Somat. Cell Molec. Gen. 27, 75-83.

17. Aurisicchio, L., Mancini, R., and Ciliberto, G. (2014). Cancer vaccination by electrogene-transfer. Expert Rev. Vaccines. 12, 1127-1137.

18. Yadav, M., Jhunjhunwala, S., Phung, Q.T., Lupardus, P., Tanguay, J., Bumbaca, S., Franci, C., Cheung, T.K., Fritsche, J., Weinschenk, T., et al. (2014). Predicting immunogenic tumour mutations by combining mass spectrometry and exome sequencing. Nature. 515, 572-576. 
19.Wang, L., Jiang, S., Deng, Z., Dedon, P.C., and Chen, S. (2019) DNA phosphorothioate modification - a new multi-functional epigenetic system in bacteria. FEMS Microbiol. Rev.43,109-122.

20. Rizzuto, G., Cappelletti, M., Mennuni, C., Wiznerowicz, M., De Martis, A., Maione, D., Ciliberto, G., La Monica, N., and Fattori, E. (2000). Electro Gene-transfer results in a high level transduction of rat skeletal muscle and corrects anaemia of renal failure. Hum. Gene Ther. 11, 1891-1900.

21. Cappelletti, M., Zampaglione, I., Rizzuto, G., Ciliberto, G., La Monica, N., and Fattori, E. (2003). Gene electro-transfer improves transduction by modifying the fate of intramuscular DNA. J. Gene Medicine. 5, 324-332.

22. Giannetti, P., Facciabene, A., La Monica, N., and Aurisicchio, L. (2006). Individual mouse analysis of the cellular immune response to tumor antigens in peripheral blood by intracellular staining for cytokines. J. Immunol. Methods. 316, 84-96.

23. Conforti, A., Peruzzi, D., Giannetti, P., Biondo, A., Ciliberto, G., La Monica, N., and Aurisicchio, L. (2009). A Novel Mouse Model for Evaluation and Prediction of HLAA2-restricted CEA Cancer Vaccine Responses. J. Immunother. 32, 744-754. Harper, L.B., Beare, C.M., Bagdon, W.J., and Nichols, W.W. (2000). Plasmid DNA vaccines: investigation of integration into host cellular DNA following intramuscular injection in mice. Intervirology. 43, 258-272.

25. Conforti, A., Marra, E., Roscilli, G., Palombo, F., Ciliberto, G., and Aurisicchio, L. (2020). Are Genetic Vaccines the Right Weapon against COVID-19? Mol. Ther. 28:1555-1556.

26. Conforti, A., Marra, E., Palombo, F., Roscilli, G., Ravà, M., Fumagalli, V., Muzi, A., Maffei, M., Luberto, L., Lione, L., et al. (2022). COVID-eVax, an electroporated DNA vaccine candidate encoding the SARS-CoV-2 RBD, elicits protective responses in animal models. Mol. Ther. 30, 311-326.

27.Guo, Y., Lei, K., and Tang, L. (2018). Neoantigen Vaccine Delivery for Personalized Anticancer Immunotherapy. Front. Immunol. 9,1499. 
28. Armengol, G., Ruiz, and L.M., Orduz, S. (2004). The injection of plasmid DNA in mouse muscle results in lifelong persistence of DNA, gene expression, and humoral response. Mol. Biotechnol. 27, 109-118.

29.Bax, R., and Green, S. (2015). Antibiotics: The changing regulatory and pharmaceutical industry paradigm. J. Antimicrob. Chemother. 70, 1281-1284.

30.Walters, A.A., Kinnear, E., Shattock, R.J., McDonald, J.U., Caproni, L.J., Porter, N., Tregoning, J.S. (2014). Comparative analysis of enzymatically produced novel linear DNA constructs with plasmids for use as DNA vaccines. Gene Ther. 21, 645652.

31.Scott, V.L., Patel, A., Villareal, D.O., Hensley, S.E., Ragwan, E., Yan, J., Sardesai, N.Y., Rothwell, P.J., Extance, J.P., Caproni, L.J., and Weiner, D.B. (2015). Novel synthetic plasmid and Doggybone DNA vaccines induce neutralizing antibodies and provide protection from lethal influenza challenge in mice. Hum. Vaccin. Immunother. 11, 1972-1982.

32. Allen, A., Wang, C., Caproni, L.J., Sugiyarto, G., Harden, E., Douglas, L.R., Duriez, P.J., Karbowniczek, K., Extance, J., Rothwell, P.J., et al. (2018). Linear doggybone DNA vaccine induces similar immunological responses to conventional plasmid DNA independently of immune recognition by TLR9 in a pre-clinical model. Cancer Immunol. Immunother. 67, 627-638.

\section{$551 \quad$ Figure Legends}

552 Figure 1. Manufacturing process of DNA amplicons. (A) A schematic representation

553 showing the assembly of termini PS-modified amplicon expression vectors via a PCR 554 device is shown. The antigen-encoding sequence, taken from a plasmid DNA, comprises

555 a promotor, one or more ORFs and a terminator. Amplicon expression vector, encoding 556 the selected sequence, is syntethized and then amplified through PS-modified PCR 
557 primers in order to produce a plurality of amplicon expression vectors. (B) A schematic 558 representation of gene expression cassettes is shown for luciferase, conTRT and M8 559 constructs. (C) HPLC chromatogram of two representative DNA amplicons, one 560 synthetized by means of Biolase ${ }^{\mathrm{TM}} \mathrm{Taq}$ (top) and one synthetized by means of MyFi ${ }^{\mathrm{TM}}$ 561 Taq (bottom). (D) Electrophoresis gel of four DNA amplicons, all encoding luciferase gene 562 (synthetized by means of Biolase ${ }^{\mathrm{TM}}$ or MyFi ${ }^{\mathrm{TM}}$ Taq polymerase, with or w/o PS-modified 563 primers).

564 Figure 2. In vivo expression of DNA amplicons. (A) Schematic representation of the 565 experimental setup. BALB/c mice were injected i.m. by DNA-EP, with equimolar 566 concentrations of plasmid DNA or DNA amplicons encoding for luciferase (50-10-5 $567 \mu \mathrm{g} / \mathrm{mouse}$ ). (B) After 1-2-7 days post DNA injection, luciferase signal was measured by 568 performing optical imaging at IVIS. Although a decreased expression was detected in 569 DNA amplicons injected mice after 7 days, no difference was measured between DNA amplicons synthetized by means of different Taq polymerase.

571 Figure 3. Assessment of in vivo DNA amplicon expression. By means of optical 572 imaging at IVIS, in vivo plasmid DNA and amplicon expression was assessed after 24h, $57348 \mathrm{~h}$ and one week post DNA injection at different DNA doses, $50 \mu \mathrm{g}(\mathbf{A})-10 \mu \mathrm{g}(\mathbf{B})-5$ $574 \mu g(\mathbf{C})$.

575 Figure 4. In vivo immunogenicity of DNA amplicons. (A) Schematic representation of 576 experimental setup. BALB/c mice were injected, by means of i.m. DNA-EP, with three 577 doses (50-10-5 $\mu \mathrm{g} / \mathrm{mouse}$ ) of a DNA plasmid, and equimolar doses of DNA amplicons, 578 both encoding for conTRT. All mice were vaccinated following a prime-boost regimen 579 (days 0 and 21) and blood analysis was performed at day 28 by intracellular cytokine 580 staining. Assessment of Th1 (B) and Th2 (C) immune response was done on PBMCs 581 isolated at day 28. Significance was determined using Mann-Whitney test, ${ }^{*} p<0,05{ }^{* *} p<$ $582 \quad 0.01$.

583 Figure 5. In vivo antitumoral effect of DNA amplicons. (A) Schematic representation 584 of experimental setup in a neoantigen cancer murine model. After tumor challenge, 
$585 \mathrm{C} 57 \mathrm{BI} / 6$ mice received a combination of DNA (plasmid or amplicon, administered at days

5862,9 and 16) and ICls ( $\alpha$ CTLA-4 or $\alpha$ PD1, administered at days 3,6 and 9). (B) Neoantigen-

587 specific $\mathrm{T}$ cell response was assessed by IFN $\gamma$ ELISpot performed on splenocytes

588 collected at day 23 and stimulated with neoantigen peptide pools. Significance was

589 determined using Mann-Whitney test, ${ }^{*} p<0,05^{* *} p<0.01$. (C) Panel depicts tumor growth

590 curve up to sacrifice at day 23. 

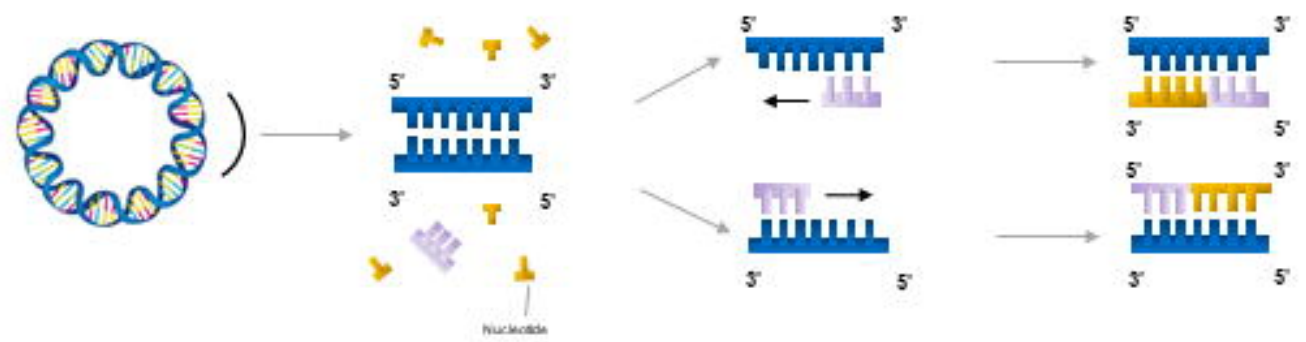

B

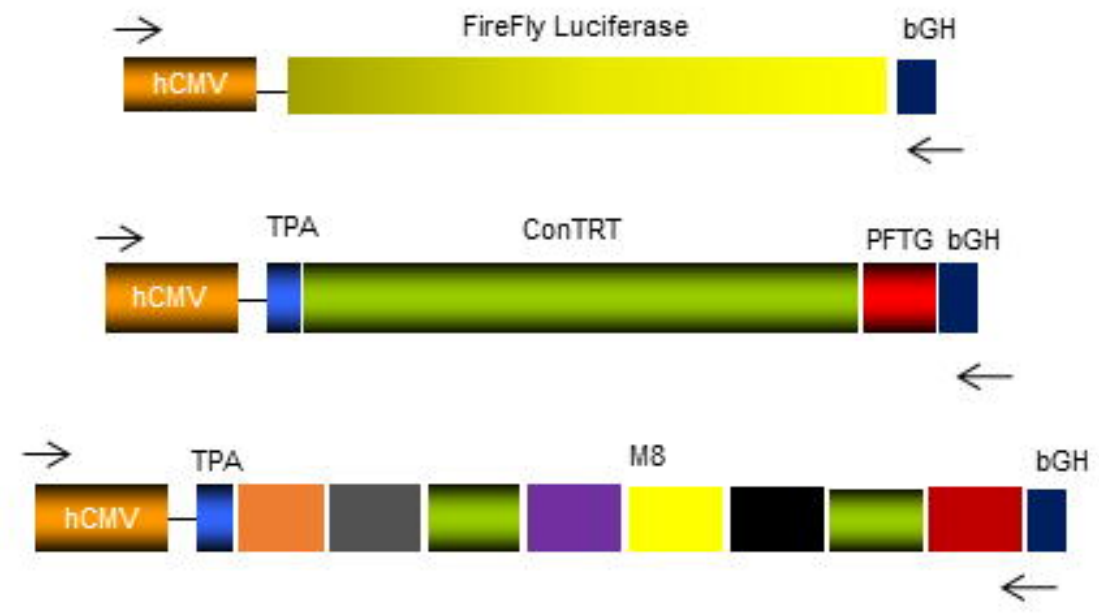

C

D
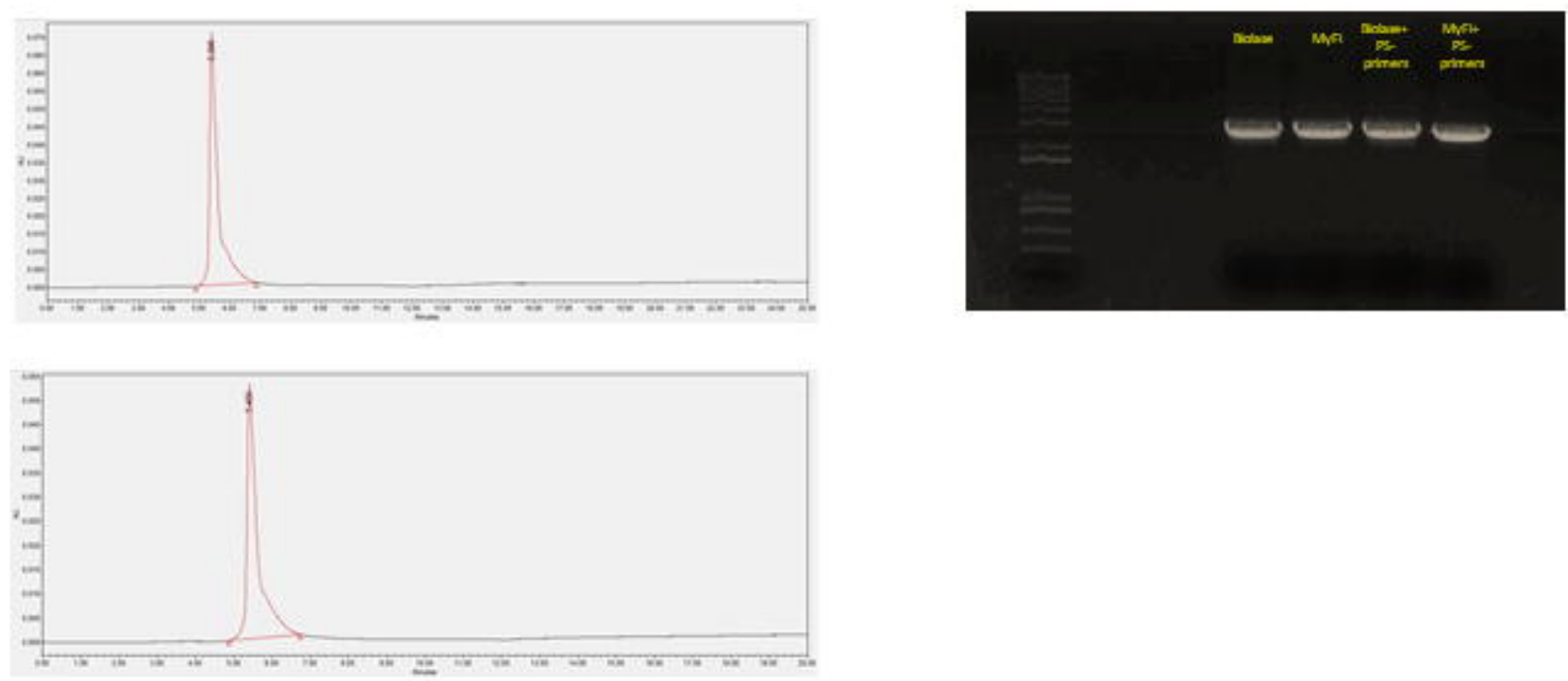
Fig.2

A
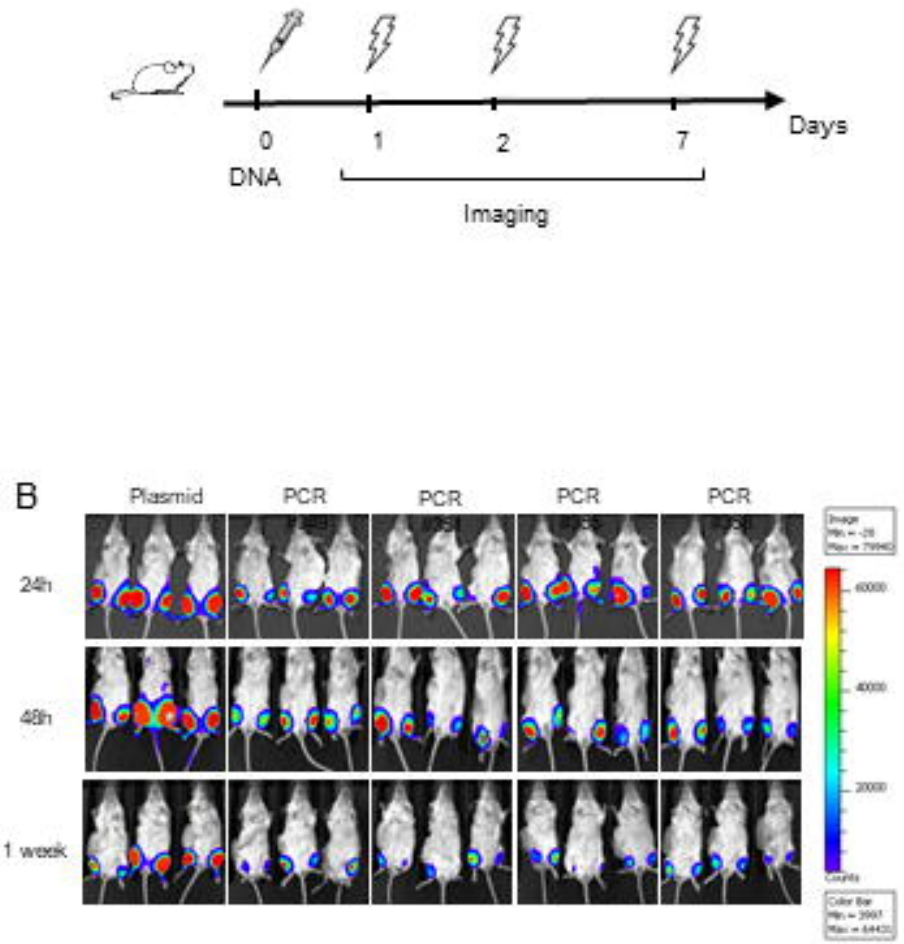
Fig.3

A

$50 \mu \mathrm{gg}$

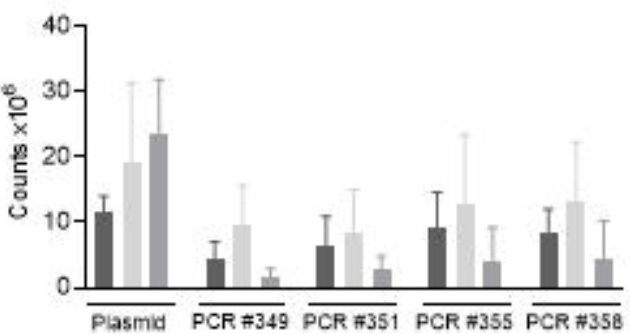

ㄹ. $24 \mathrm{~h}$

$48 \mathrm{~h}$

$1 \mathrm{w}$

B
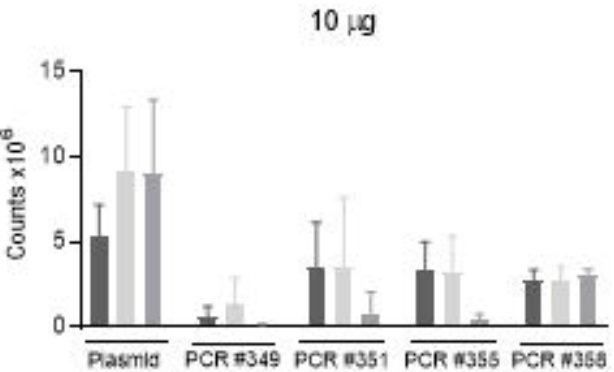

$24 \mathrm{~h}$

핌 $48 \mathrm{~h}$

- $1 \mathrm{w}$

C

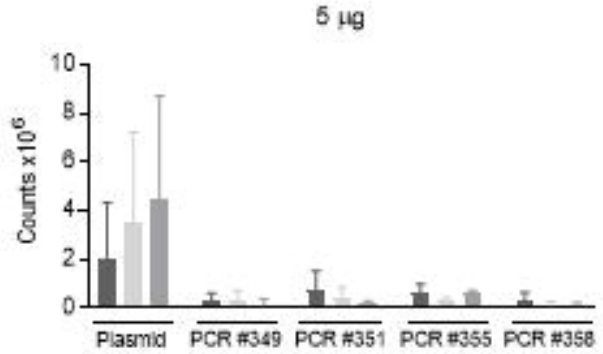

- $24 \mathrm{~h}$

$48 \mathrm{~h}$

a $1 \mathrm{w}$ 


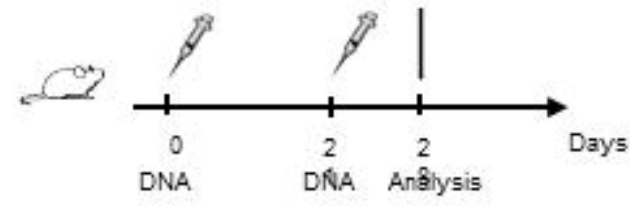

B
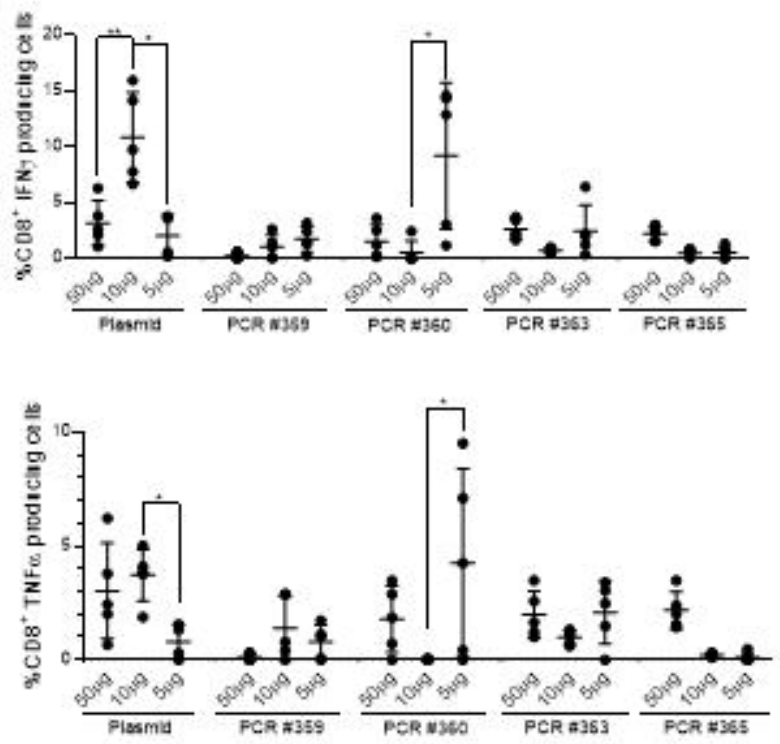

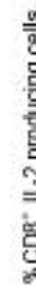

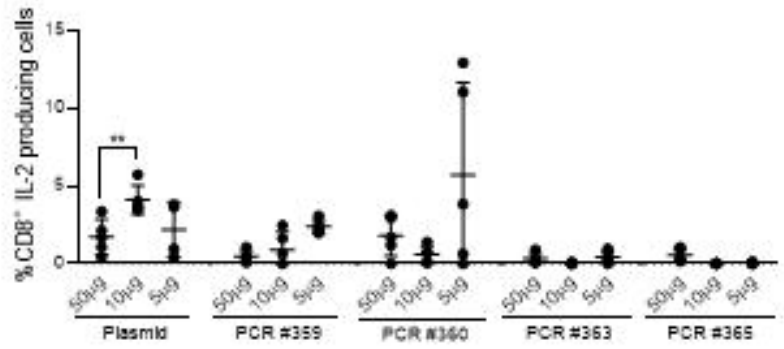

C
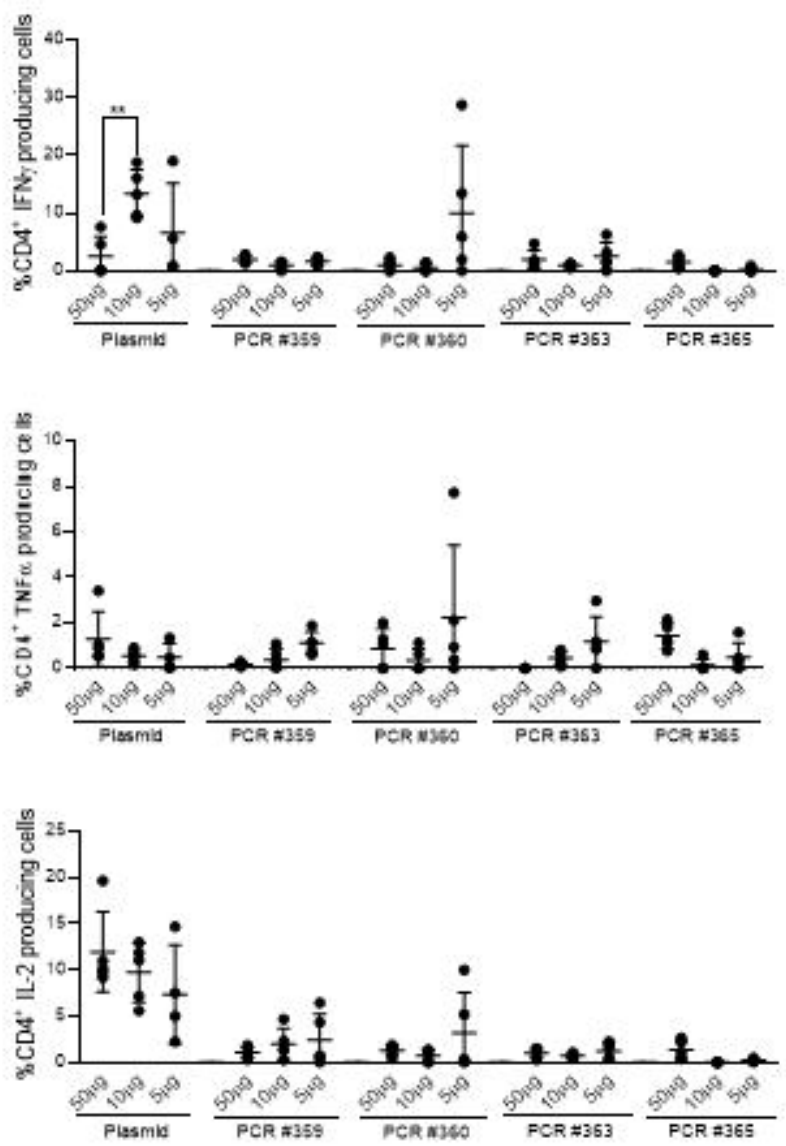
A

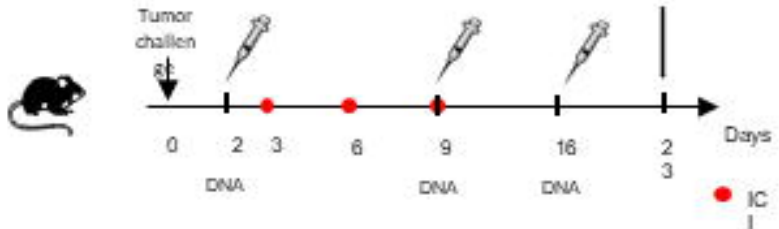

B

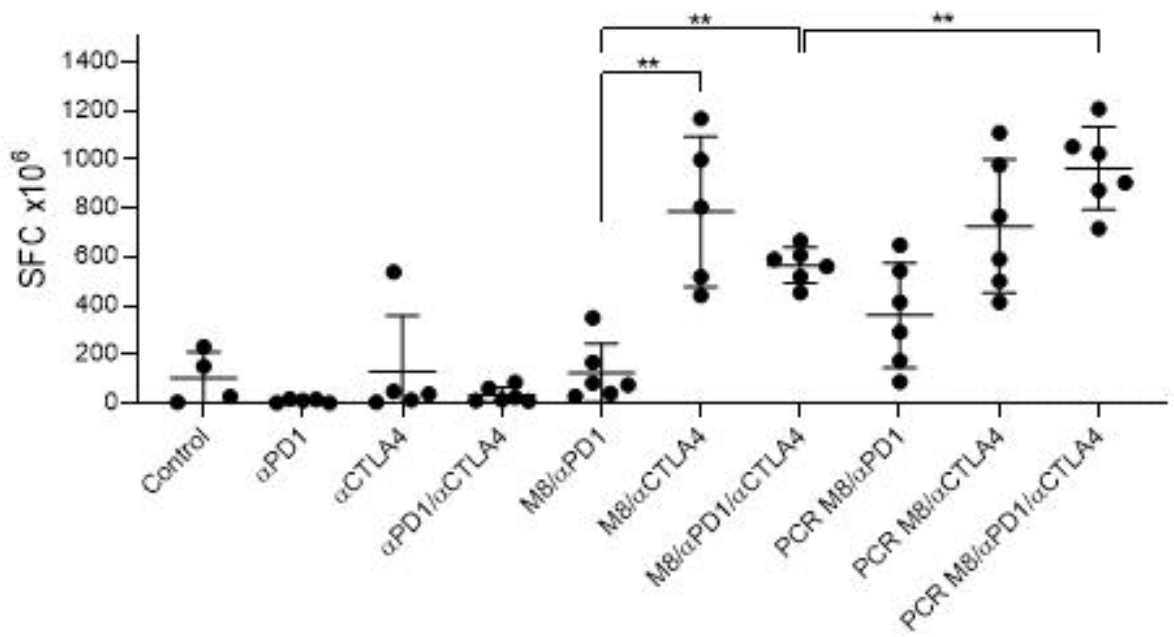

C

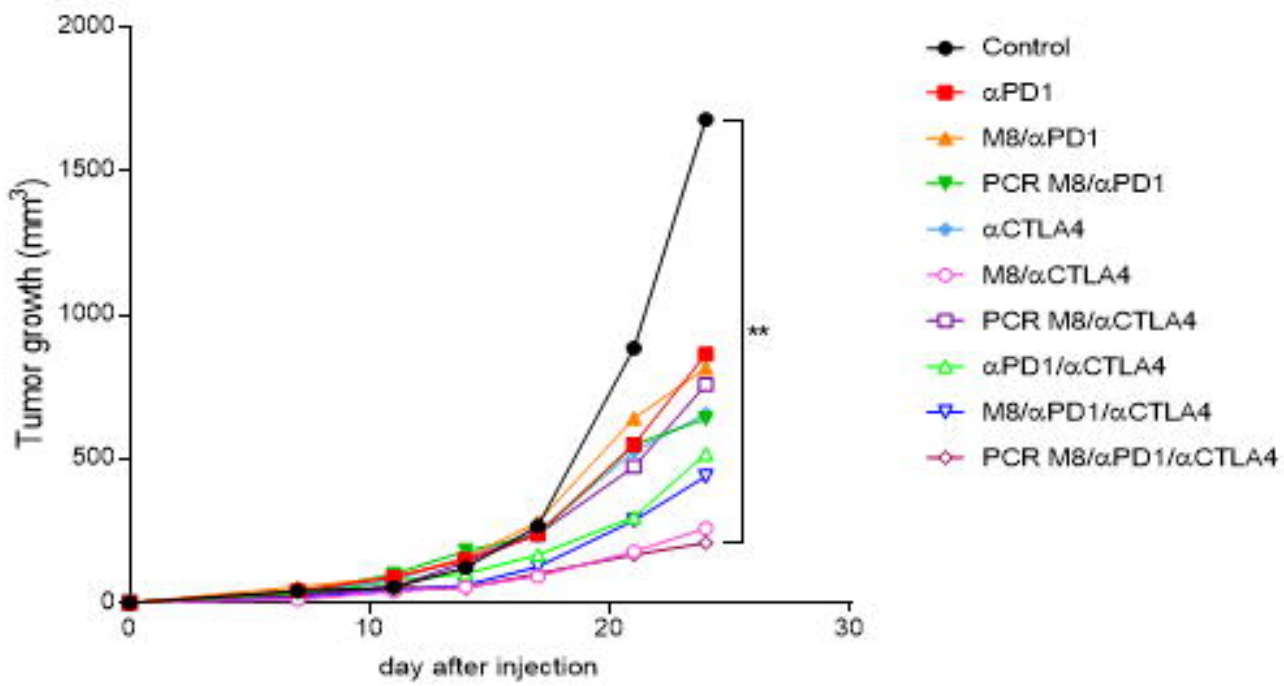

